\title{
Spectrum Usage Forecasting Model for Cognitive Radio Networks
}

\author{
Wei Yang ${ }^{1,2}$, Xiaojun Jing ${ }^{1,2}$, Hai Huang ${ }^{1,2}$ \\ ${ }^{1}$ School of Information and Communication Engineering, Beijing University of Posts and Telecommunications, \\ Beijing, 100876, China \\ [e-mail: yangweibupt@bupt.edu.cn] \\ ${ }^{2}$ Key Laboratory of Trustworthy Distributed Computing and Service, Beijing University of Posts and \\ Telecommunications, Ministry of Education \\ *Corresponding author: Wei Yang
}

Received September 12, 2017; revised October 15, 2017; accepted November 2, 2017; published April 30, 2018

\begin{abstract}
Spectrum reuse has attracted much concern of researchers and scientists, however, the dynamic spectrum access is challenging, since an individual secondary user usually just has limited sensing abilities. One key insight is that spectrum usage forecasting among secondary users, this inspiration enables users to obtain more informed spectrum opportunities. Therefore, spectrum usage forecasting is vital to cognitive radio networks (CRNs). With this insight, a spectrum usage forecasting model for the occurrence of primary users prediction is derived in this paper. The proposed model is based on auto regressive enhanced primary user emergence reasoning (AR-PUER), which combines linear prediction and primary user emergence reasoning. Historical samples are selected to train the spectrum usage forecasting model in order to capture the current distinction pattern of primary users. The proposed scheme does not require the knowledge of signal or of noise power. To verify the performance of proposed spectrum usage forecasting model, we apply it to the data during the past two months, and then compare it with some other sensing techniques. The simulation results demonstrate that the spectrum usage forecasting model is effective and generates the most accurate prediction of primary users occasion in several cases.
\end{abstract}

Keywords: Dynamic spectrum access, spectrum sensing, AR-PUER, CRNs 


\section{Introduction}

$\mathbf{W}_{\text {ith the express development of wireless communication system, it posed a tough }}$ requirement for the limited radio spectrum resource. On the other hand, in connection with the spectrum report conducted by the Federal Communications Commission (FCC), the majority of the radio spectrum is not in use in reality [1]. Therefore, the perception of CRNs has been proposed as a hopeful technology to deal with the spectrum scarcity as well as the spectrum underutilization problem [2].

The merely consideration is that the secondary users have to vacate the channel within a certain amount of time whenever the primary user becomes active. Thus, the cognitive radio network faces the tricky challenge of detecting the presence of the primary user, particularly in a low signal-to-noise ratio (SNR) region, since the signal of the primary user might be severely alleviated due to multipath and shadowing before reaching the secondary user. To ensure that there will be no harmful intervention to the primary user, the secondary users need to sporadically detect the presence of the primary user. There are several factors that avoid the spectrum sensing from operating in a reliable manner. One factor is that the strength of the primary users' signals could be very weak when they reach the secondary users. If the secondary user makes a defective decision in detection and establishes transmitting when the primary users are active, its own signal will meddle with the primary users' signals. In cognitive radio networks (CRNs), one aim is to prevent the primary users from the case that the secondary users utilize the primaty users' spectrum for opportunistic wireless transmissions while the spectrum was being used. To gurantee the spectrum usage of primary users, secondary users are required to sense and check the appearence of primary users. On the other hand, the achievable throughput for the secondary users has also considered in reality.

The performance of spectrum sensing was evaluated by the detection probability and the probability of false alarm [3]. Fail notice in detection will lead to interference with primary users and false alarm will reduce the spectral efficiency [4]. The optimal detection performance occurs in the situation that the detection probability is maximized subject to the constraint of the false alarm probability.

The core technology behind spectrum reuse in CRNs is spectrum sensing, however, the dynamic and unpredictable sensing environment largely affect the detection efficiency, which bring about poor performance in the protection of primary users.

\section{Related Work}

After suvey the literature of spectrum sensing scheme, a number of algorithms have been proposed to identify the presence of primary users. The most popular spectrum sensing methods that have been proposed are energy detection [5], cyclostationary feature detection [6] and matched-filtering detection [7]. Some other examples of the existing proposals include covariance-based detection [8]. Matched filtering works based on the prior knowledge of the signal and needs a well time synchronization. Although the covariance-based detection based method performs much better than the other methods, its required sampling frequency is much higher than the normal condition which leads to a higher complexity of implementation.

Energy detection is a classical blind spectrum sensing format without the prior knowledge of signal, time synchronization and a higher sampling frequency. However, the main drawback is noise uncertainty which incurred by the estimation of noise variance, especially 
under a lower signal-noise-ratio (SNR). For the sake of noise uncertainty removal, plenty of corresponding schemes are proposed, such as cooperative spectrum sensing [9].

As a useful tool for improving the user's quality of experience, delay announcement has received substantial attention recently [10]. Basically, introducing the user response to the data-driven delay estimation yields two main challenges: First, user response is a subjective metric, and it is intractable to provide a uniform mathematic expression. Second, the interaction between the user response and waiting time is also very difficult to capture [11]. Nevertheless, majority of energy detection based methods are devoted to remove noise uncertainty while the effect is not efficient enough. In the large extend, noise uncertainty and other interference from sensing environment determines the accuracy of estimation that whether the given spectrum is used or not. Therefore, it is vital to remove the interference for the enhancement of detection accuracy and the applicability of sensing scenarios. In the context of cognitive radio netwoks, close attention has been paid. Andrea Mariani etc. explored the SNR wall phenomenon caused by uncertain noise for energy detection based method, addressing the threshold design and giving the conditions for the existence of the SNR wall. Valentin Rakovic etc. proposed an optimization approach for cooperative spectrum sensing utilizing energy detection with estimated noise power.

As a result, a spectrum usage forecasting model is proposed in this paper on the basis of auto regressive enhanced primary user emergence reasoning (AR-PUER). The proposed model performs much better than the commonly used methods. Moreover, unlike the energy detector [12] and prior cyclostationary methods, the novel AR-PUER model here is blind and does not require information about the multipath channel distortions that the primary users has undergone on its way to reaching the secondary user. Besides, the model also does not suffer from the noise uncertainty and other interference which may lead to poor performance in the detection of spectrum usage. Due to not affected by the noise uncertainty and environmental interference, the AR-PUER model's detection capability will be greatly heightened for the decrease of background noise.

The rest of this paper is organized as follows. In Section 3, the auto regressive enhanced primary user emergence reasoning (AR-PUER) model is presented for the proposed scheme, which includes the detection method, primary user behavior characteristic and AR-PUER treatment process. In Section 4, the performance of proposed approach is analyzed, and numerical results are presented. Finally, section 5 briefly concludes this paper.

\section{System Model}

In this section, we consider fundamental CRNs. Let $x(t)$ stands for the signals received at the secondary user, while $s(t)$ and $n(t)$ denote the signal transmitted by primary users respectively. Generally speaking, $x(t)$ can be briefly classified into two basic categories.

$$
\left\{\begin{array}{cc}
x(t)=n(t) & H_{0} \\
x(t)=s(t)+n(t) & H_{1}
\end{array}\right.
$$

where $H_{0}$ and $H_{1}$ represent the absence and the presence of primary user's signal. For primary user's signal detection, the detection probability $P_{d}$ and the false alarm probability $P_{f}$ can be defined as 


$$
\left\{\begin{array}{l}
P_{d}=P_{r}\left\{H_{1} \mid H_{1}\right\} \\
P_{f}=P_{r}\left\{H_{1} \mid H_{0}\right\}
\end{array}\right.
$$

It is assumed that all the secondary users experience an independent and identically distributed fading. The sensors are conditionally independent, which means that the secondary user's measurements are independent, but for each secondary user, the same hypothesis $\left\{H_{0}, H_{1}\right\}$ applies.

\subsection{Time Correlation Estimate}

In order to find out the spectrum usage of primary users, the concept of time division is introduced. Time division belongs to time interval which based on a statistical analysis of the primary users' spectrum over a period of time. For some primary users, they may have more than one time division.

In order to make the consequence more accurate, we will compensate the error through statistical approach. With the intention of evaluate the disparity between the primary users' spectrum access time and average time, the concept of time similarity is proposed, which can be defined as

$$
\tau(\alpha, \beta)=1-\delta(\alpha, \beta)
$$

where $\delta(\alpha, \beta)=\frac{|\alpha-\beta|}{\varepsilon}, \xi=|\alpha-\beta|$.

In (3), $\alpha$ indicates the initiation spectrum access time which independent from the statistical time of primary users, $\beta$ stands for the average time that primary users have accessed to the spectrum, while $\varepsilon$ shows the time is twenty-four hours. For the meantime, we classify $\xi$ as time freeness which has two kinds of values:

1) Primary users' own actual time $\xi^{\prime}$ is known as the code value of time freeness; in the same way, $\tau\left(\xi^{\prime}\right)$ is the code value of time similarity.

2) According to $\xi=|\alpha-\beta|$, we can calculate the observation value of time freeness $\xi$, while the observation value of time similarity is $\tau(\xi)$ or $\tau(\alpha, \beta)$.

The code value of time similarity can be briefly classified into two intervals $\left(\tau\left(\xi^{\prime}\right), 1\right)$ and $\left(0, \tau\left(\xi^{\prime}\right)\right)$, when the observation value $\tau(\alpha, \beta)$ is valid in $\left(\tau\left(\xi^{\prime}\right), 1\right)$, accordingly we call $\left(\tau\left(\xi^{\prime}\right), 1\right)$ as the valid interval; as a result, $\left(0, \tau\left(\xi^{\prime}\right)\right)$ is the invalid interval.

Considering the situation that a primary user maybe repeated access to the spectrum, therefore, the primary user exist multiple time similarity, that is to say, there is more than one time similarity located in the valid interval. Consequently, the time similarity of primary user A at $t_{1}, t_{2}, \cdots, t_{k}, \cdots$ can indicated as $\tau_{1}\left|\begin{array}{l}A \\ t_{1}\end{array}, \tau_{2}\right| \begin{aligned} & A \\ & t_{2}\end{aligned}, \cdots, \tau_{k} \mid \begin{aligned} & A \\ & t_{k}\end{aligned}, \cdots$ respectively. Analogously, we see that $\left.\tau_{1}\left(\alpha, t_{1}\right)\right|_{t_{1}} ^{A},\left.\tau_{2}\left(\alpha, t_{2}\right)\right|_{t_{2}} ^{A}, \cdots,\left.\tau_{k}\left(\alpha, t_{k}\right)\right|_{t_{k}} ^{A}, \cdots$. In the valid interval, the maximum time similarity can characterized as $\left.\tau_{\max }\left(\alpha, t_{i}\right)\right|_{t_{i}} ^{A}$, while the minimum time similarity can indicated 
as $\left.\tau_{\text {min }}\left(\alpha, t_{j}\right)\right|_{t_{j}} ^{A}$. Hence, they can illustrated as $\tau_{\text {max }} \mid \begin{aligned} & A \\ & t_{i}\end{aligned}$ and $\tau_{\text {min }} \mid \begin{aligned} & A \\ & t_{j}\end{aligned}$ for short.

The above time similarity in which interval was valid is mainly determined by the time freeness $\xi^{\prime}$, because the standard deviation is the best way to evaluate time fluctuations, we can define its standard deviation as the time freeness $\xi^{\prime}$, which implies

$$
\xi^{\prime}=\sqrt{\frac{1}{N} \sum_{i=1}^{N}\left(x_{i}-\bar{x}\right)^{2}}
$$

When $\xi^{\prime}$ is little, the time similarity $\tau$ will be relatively large, and the effective interval will be narrowed accordingly, which reveal the primary users' spectrum access behavior has a strong regularity. On the contrary, when $\xi^{\prime}$ is large, the time similarity $\tau$ will be little at once, and the effective interval will be broaden, which expose the primary users' spectrum access behavior has a weak regularity.

\subsection{Primary User Behavior Characteristic}

At first, we define a fixed number of days $(m)$ as criteria. In order to minimize the interference of human factors, the maximum allowed range of time periods should not exceed the code value of time freeness. The primary users' spectrum access duration begin with $D$, and end up with $D+m$, which located in the area from line $R$ to $R+n$, with the maximum trigger value during the time period $R+n$.

The access behavior employs natural number coding, that is, for the access behavior which length is $n$, each of them take an integer value from 1 to $m$ (the maximum value). The access behavior is constructed as follows

\begin{tabular}{|l|l|l|l|}
\hline $\mathrm{R}$ & $\mathrm{n}$ & $\mathrm{D}$ & $\mathrm{m}$ \\
\hline
\end{tabular}

In the process of operation, we need to determine the evaluation of each primary user. The evaluation can be calculated on the basis of assessment function

$$
f_{k}=\sum_{e \in P} \omega(e)
$$

where $f_{k}$ is the evaluation of primary user $k, e$ is the unit grid, $P$ is the enclosed area with primary users' access behavior, and $\omega(e)$ is the value of unit.

In the progress for the primary users' access behavior characteristic, the procedure can be briefly expressed as follows:

1) Setup the initial number of days as $P(0)$ after initialization;

2) Evaluate the primary users in accordance with the former assessment function (5), and calculate the evaluation value of primary users in $P(t)$;

3) Carry on interleaved computation, we can obtain $P(t+1)$ from $P(t)$ after the mean value calculation;

4) Calculate the end conditions, if $t \leq T$, then $t \rightarrow t+1$, and next go to step 2); if $t>T$, then output the primary user with maximum time similarity $\left.\tau_{\max }\right|_{t_{i}} ^{A}$ as the optimal solution, and stop the calculation. 


\subsection{Proposed AR-PUER Model}

The spectrum usage forecasting model in this paper is based on linear prediction and case based reasoning $[13,14]$, which involves predicting a future value of a stationary discrete-time stochastic process, given a set of past samples of the process.

Considering the time series of received signal vectors as $\left\{W_{t}, W_{t-1}, W_{t-2}, \ldots, W_{t-p}\right\}$. In the auto regressive $A R(p)$ model, samples $\left\{W_{t}, W_{t-1}, W_{t-2}, \ldots, W_{t-p}\right\}$ are used to predict the condition of primary user $a_{t}$. In this paper, forward linear prediction will be used. The forward prediction at time $t$ is denoted by $a_{t}$ and is given by

$$
W_{t}-\varphi_{1} W_{t-1}-\varphi_{2} W_{t-2}-\cdots-\varphi_{p} W_{t-p}=a_{t}, t=0, \pm 1, \pm 2, \cdots
$$

The retardation factor $B$ is an operator, which have the arithmetic operation $B^{k} W_{t}=W_{t-k}, k \geq 1$. The stipulation of primary user $a_{t}$ is then defined as

$$
\left(1-\varphi_{1} B-\varphi_{2} B^{2}-\cdots-\varphi_{p} B^{p}\right) \cdot W_{t}=a_{t}
$$

When $\Phi(B)=1-\varphi_{1} B-\varphi_{2} B^{2}-\cdots-\varphi_{p} B^{p}$, the mentioned formula above can illustrated as

$$
\Phi(B) \cdot W_{t}=a_{t}, t=0, \pm 1, \pm 2, \cdots
$$

The proposed model in this paper combines linear prediction with the received primary users emergence reasoning data. Auto regressive scheme is a widely used technique in numerical analysis and linear algebra [15]. Based on this approach, some signal statistics will be defined, which reveal distinctive features only in the presence of primary users. This discrimination will improve the probability of detection.

In the function $W_{t}=\Phi^{-1}(B) \cdot a_{t}$, in which $G(B)=\Phi^{-1}(B)=\sum_{k=0}^{\infty} G_{k} B^{k},|B|<1$, we see that $W_{t}=\sum_{k=0}^{\infty} G_{k} a_{t-k}$ where $G_{k}, k=0,1,2, \cdots$ is the Green's function of the $A R(p)$ model. Analogously, in $a_{t}=\Phi(B) \cdot W_{t}$ where $I(B)=\Phi(B)=G^{-1}(B)=I_{0}-\sum_{k=1}^{\infty} I_{k} B^{k},|B|<1$ ( $I_{0}=1$ ), we can obtain $a_{t}=W_{t}-\sum_{k=1}^{\infty} I_{k} W_{t-k}$. It can be shown from the function that $I_{k}, k=0,1,2, \cdots$ is the inverse function for $A R(p)$.

From the stationary sequence $\left\{W_{t}\right\}$, which $W_{k}, W_{k-1}, W_{k-2}, \cdots$ was known, we can have $\hat{W}_{j}=W_{j}(j \leq k), \hat{a}_{k+l}=0(l \geq 1)$. In doing so, some signal statistics forecast formulas based on the auto regressive model will be obtained below.

$$
\begin{gathered}
\hat{W}_{k}(l)=\varphi_{1} \hat{W}_{k}(l-1)+\varphi_{2} \hat{W}_{k}(l-2)+\cdots+\varphi_{p} \hat{W}_{k}(l-p), l>q \\
\hat{W}_{k}(l)=\sum_{j=1}^{\infty} I_{j}^{(l)} \cdot W_{k+1-j}, \quad l \geq 1
\end{gathered}
$$


where $\left\{\begin{array}{cc}I_{j}^{(1)}=I_{j} & j \geq 1 \\ I_{j}^{(l)}=I_{j+l-1}+\sum_{m=1}^{l-1} I_{m} I_{j}^{(l-m)} & j \geq 1, \quad l \geq 2\end{array}\right.$.

Since the primary user emergence reasoning method shares the same training samples and procedures with the auto regressive manner, it is possible to combine the training samples, and improve the forecasting accuracy with near vectors in both temporal and feature space. Thus we propose an spectrum usage forecasting model to enhance the precision of primary user presence without requiring the information about the channel. The spectrum access behavior reasoning model of primary users is shown in Fig. 1.

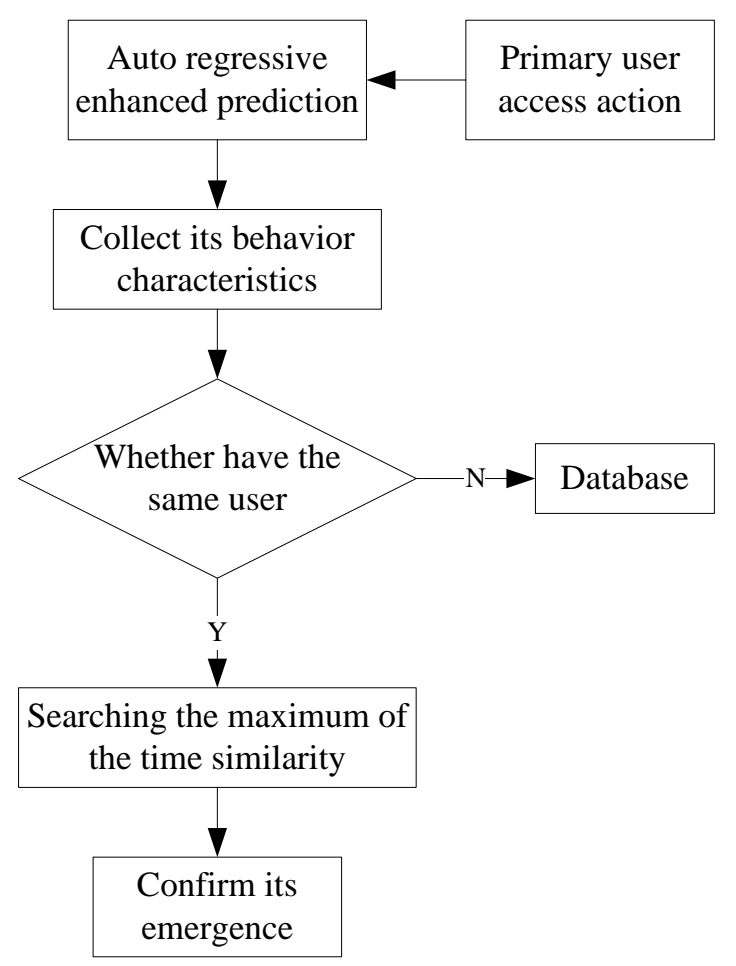

Fig. 1. Spectrum usage forecasting model

For the spectrum usage forecasting model training with historical data, the procedures of the combined AR and PUER scheme are as follows:

First of all, searching all the users in the stored access behavior, which have the similar spectrum access time with the real primary user, and then utilize the auto regressive model to enhance the prediction of primary users. Moreover, collect its access behavior characteristics and analysis to determine the primary users which in accordance with the daily access habits according to the time similarity. Once again, chase down its nearest neighbor by means of the above mentioned primary user, and then calculate the time span of them. Furthermore, update the searched user in the light of its access behavior and correction rule. Eventually, make a review and revision, thus evaluate the necessity whether hold it back. 
As to the situation that primary users may have a delay during the spectrum access procedure because of some special circumstance, which will result in $\tau(\alpha, \beta)$ is invalid. Accordingly, we introduce the conception of behavior similarity. Frequently, the primary user's construction set is consist of feature set and relation set, which can indicated as $U: U=\{$ Feature, Relation $\}$. Besides, the configuration set $U$ can also include some other user's properties, for example, with the primary user's different access behavior, it may contain the weight coefficient $W$, that is to say, $U: U=\{$ Feature, Relation, Weight $\}$.

Supposing that User 1 and User 2 match all the characteristics, then we have $U_{1}=U_{2}$. If they only have some characteristic values in common, they are partial similarity, and we can utilize Sim to express the similarity between them. It can also be defined as the ratio between the matched characteristics with all the features, that is to say $\operatorname{Sim} \in[0,1]$. The larger the relation value, the higher the similarity between them. When $\operatorname{Sim}=1$, they are the same user; while different users when $\operatorname{Sim}=0$. The assemblage of any two users can be defined as $V_{A}=\left\{a_{1}, a_{2}, \cdots, a_{n}\right\}, V_{B}=\left\{b_{1}, b_{2}, \cdots, b_{n}\right\}$, and then the similarity between them can be expressed as

$$
\operatorname{Sim}(A, B)=\frac{1}{n} \sum_{i=1}^{n} \sin \left(a_{i}, b_{i}\right)
$$

If the user's access behavior is not stable, we can set the corresponding weight. Consequently, the expression of behavioral similarity, which include the weight coefficient, can represented as

$$
\operatorname{Sim}(A, B)=\frac{\sum_{i=1}^{n} \sin \left(a_{i}, b_{i}\right) w_{i}}{\sum_{i=1}^{n} w_{i}}
$$

where $\sin \left(a_{i}, b_{i}\right)=\left\{\begin{array}{l}1, a_{i}=b_{i} \\ 0, a_{i} \neq b_{i}\end{array}\right.$, and $\alpha_{i}$ stands for the accessed primary user, while $\beta_{i}$ express the stored primary user access action. At the moment of $a_{i}=b_{i}$, the forecast result is true, moreover, $\sin \left(a_{i}, b_{i}\right)=1$; conversely, $\sin \left(a_{i}, b_{i}\right)=0$. Simultaneously, we can define the weight coefficient $w_{i}$ flexibility on the basis of the actual usage condition.

\section{Simulations and Analysis}

Simulation experiments based on matlab platform are made to check the detection performance in this section. The radio frequency band of the very high frequency is taken for the registered band waiting for the opportunistic access of secondary users. Consequently, the carrier frequency $f_{c}$ is set as $1.0 \times 10^{8} \mathrm{~Hz}$ and the sampling frequency $f_{s}$ of secondary users is $3.0 \times 10^{8} \mathrm{~Hz}$. Considering the sensing time and detection performance simultaneously, $N=100$. 
The performance of spectrum sensing is characterized by the probability of detection $P_{d}$ and the probability of false alarm $P_{f}$. In the case, the secondary users may cause a severe interference to the primary user. On the contrary, the probability of false alarm $P_{f}$ means the probability that the secondary user judges primary user to be detected even though the primary user does not occur. Besides, the consumption of radio resource becomes worse because the secondary user changes the currently used channel into another available channel. It has been recognized that there is a tradeoff between the probability of detection and the probability of false alarm according to the sensing threshold $[16,17]$.

Since the proposed model is a combination of AR model and PUER model, each of these models are also separately utilized. The training window size is mainly set to catch more similar pattern in the historical data. Since the combined AR-PUER model is used for primary user presence forecasting, the training window can be more time-correlated by setting two months of primary user data for training.

Taking into account the condition that spectrum access of primary users at the weekend and weekday may be different; however, the overall usage of the primary users is similar. Therefore, the experiments only consider the working days, and exploit different numbers to represent various primary users. Subsequently, the simulation is carried out on the trial platform to analyze whether the AR-PUER model is effective. Through the experiment, we can get the statistics data of primary users' presence time and the model successful forecasting number of times. As shown in Fig. 2, where the "Whole" represents the total spectrum access number of primary users, while the "Success" means that the success predictive number for secondary user through the AR-PUER model. Through the data of past eight weeks, we can see the successful rate is increasing week by week, this can reflect the spectrum usage forecasting model's learning ability as time went on.

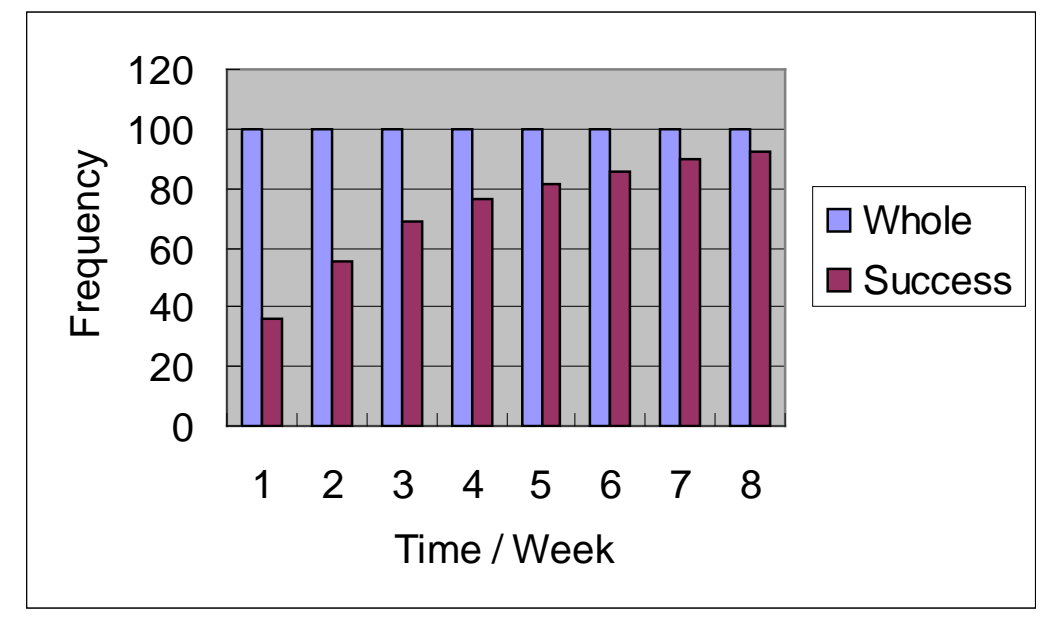

Fig. 2. Service chart of AR-PUER model

For the secondary user, the dynamic spectrum access successful implementation depends largely on the model of spectrum usage forecasting detection algorithm and the intelligent degree of the model mainly reflects in how many times it can successfully predict the primary 
users' emergence. That is to say, the more service time provided by the spectrum usage forecasting model is just the number of services that the secondary user would like to get, the higher successful forecasting rate it is. Additionally, the spectrum usage forecasting model's intelligence level is also improved and vice versa. The goal of the spectrum sensing scheme is to maximizing the probability of detection $P_{d}$ for a specific probability of false alarm $P_{f}$. Performance of a detector can be evaluated via the receiver operating characteristic (ROC) curve, in which probability of detection $P_{d}$ is drawn in terms of probability of false alarm $P_{f}$.

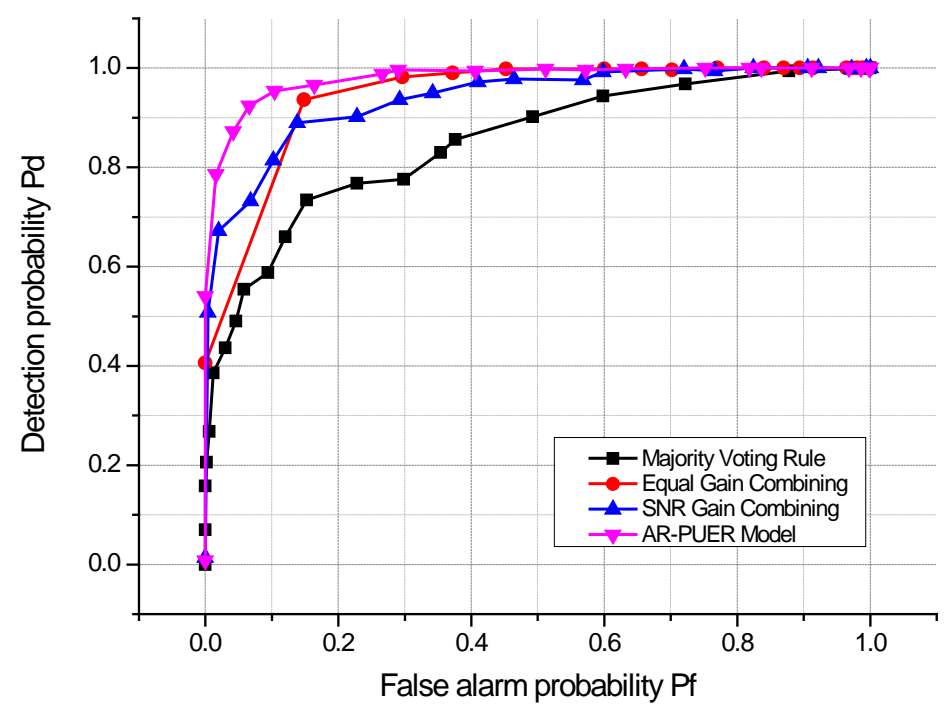

Fig. 3. Detection performance evaluation in AWGN channel

Fig. 3 Fig. 5 displays the ROC curves of several processes for spectrum sensing under various channels. Obviously, the detection performance of spectrum usage forecasting model increases about $20 \%$ on average for a given probability of false alarm compared with the classical majority voting rule. Moreover, it is also superior to the equal gain combining method and even has much advantage over the SNR gain combing fusion scheme. In summary, the classical majority voting based method always suffers from noise uncertainty, even for the relevant improved methods, resulting in a poor detection performance under strong noise conditions. However, the spectrum usage forecasting model performs spectrum sensing through the auto regressive with primary user emergence reasoning, which does not depend on the estimation of noise variance and dramatically clears the influence form noise uncertainty. Additionally, the consideration of the correlation between auto regressive and primary user emergence reasoning makes the detection of idle spectrum more accurate. As a result, the detection performance is greatly improved in return. 


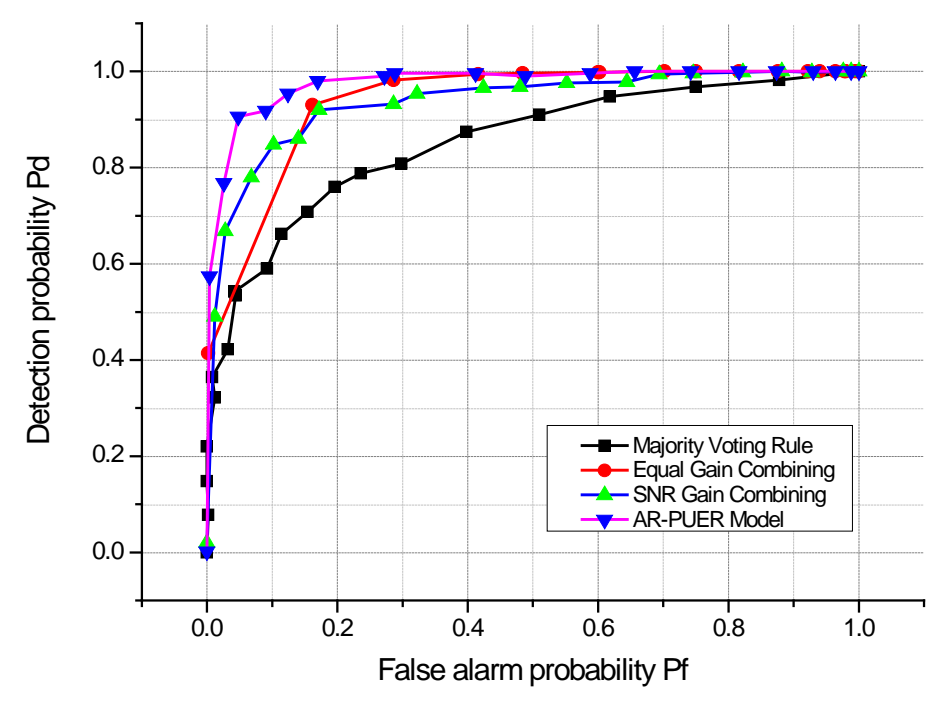

Fig. 4. ROC curves comparison of different schemes in Rayleigh channel

The major concern of any proposed algorithm is the complexity-performance tradeoff, how much we should trade the complexity to get better performance. The computational complexity of the proposed format is approximately as the conventional method; nevertheless, the increase in detection performance reaches about two times as majority voting rule at the probability of false alarm $P_{f}=0.05$ in Fig. 4, which is the common rated value for useful CRNs.

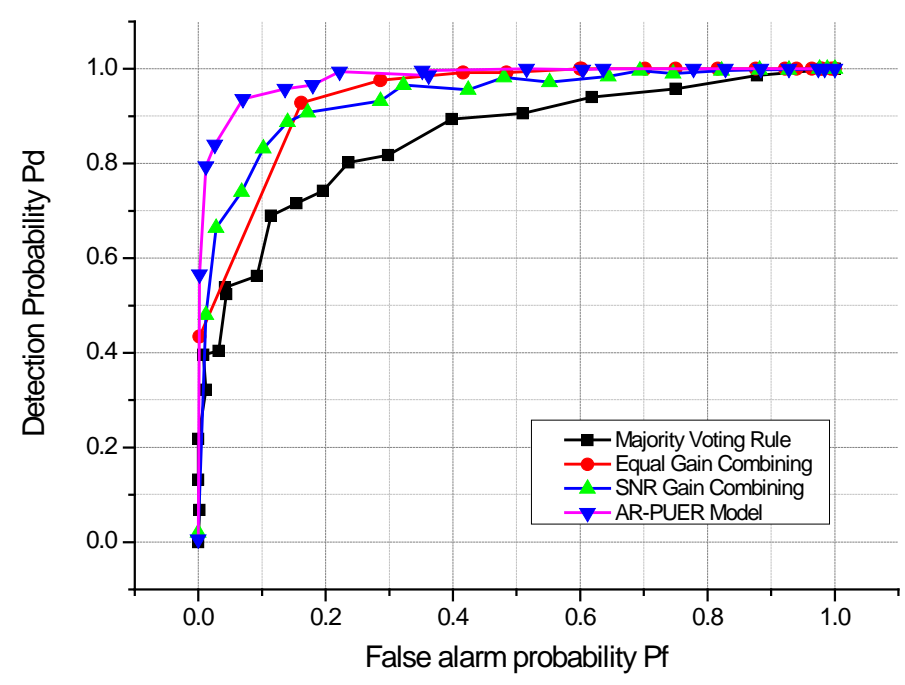

Fig. 5. Detection performance assessment in Rician channel 
Primary users in the daily use of the spectrum often occurs in the following situations, such as one day occupy multiple spectrum bands, the same primary user during different time periods of the spectrum is not the same, some spectrum resources even have not been used. Even though there are circumstances exist as above-mentioned, AR-PUER model is still more effective in providing the spectrum access prediction services for secondary users. After analysis and calculation, the success rate of AR-PUER model during the past three months are improved dramatically at the initial time, after that it tends to smooth stably, this can be seen from the data in Fig. 6.

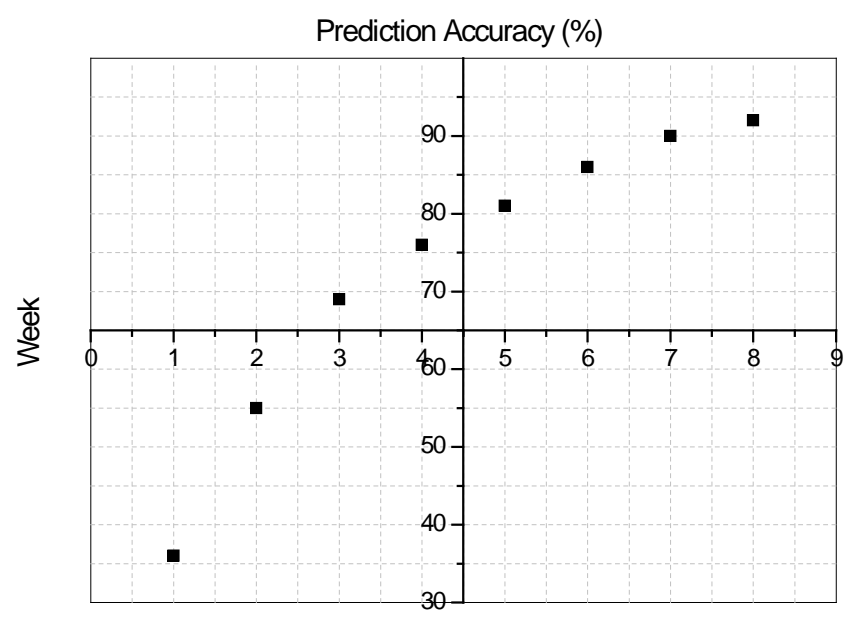

Fig. 6. Prediction trend chart of AR-PUER model

Through the data of first four weeks, it can be seen that the successful rate of the model in predicting the presence of primary users began to rise as time goes on, this phenomenon reflect the learning ability of the algorithm increases gradually. During the following four weeks, data shows that through a long time of learning, the spectrum usage forecasting model can provide stable and high quality service for secondary users, which also demonstrate that the model is effective in the implementation of dynamic spectrum access in secondary users. The whole process can be illustrated as a 3D color pie chart in Fig. 7, it can be clealy noticed that the first week's sucessful forecast rate only takes up a small part of the whole process, while the last week shares the most percentage in the sucessful prediction of primary users spectrum usage. 


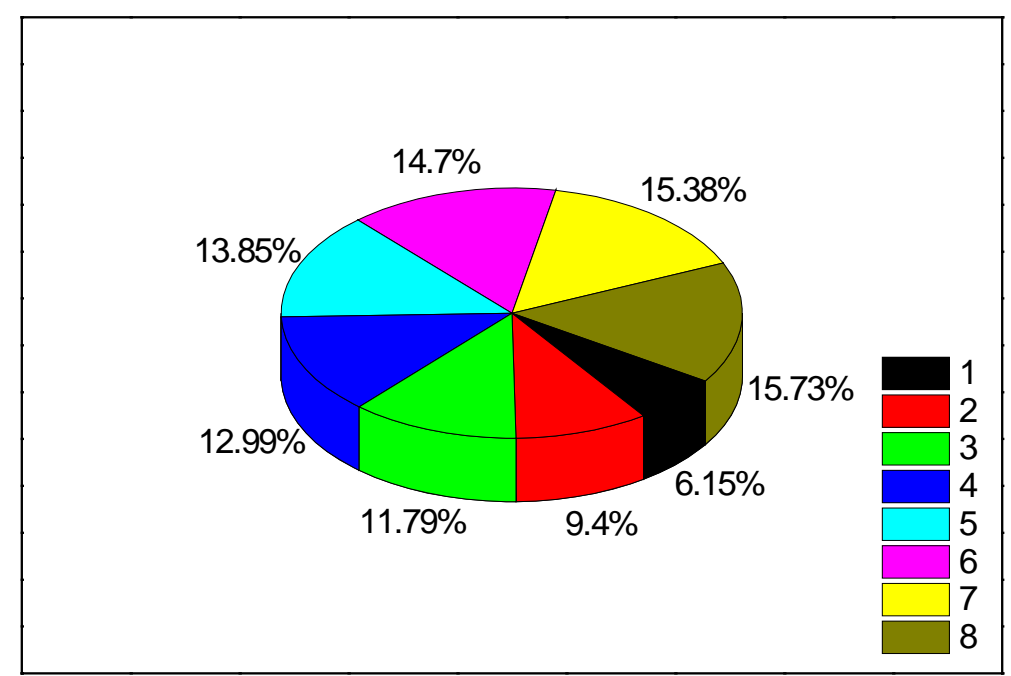

Fig. 7. 3D color pie chart of the prediction outcome

The numerical experiment illustrated that the forecasting performance was improved by combining the training samples, and the improvement was repeatable for different primary user data. Accordingly, the proposed spectrum usage forecasting model could improve the probability of detection in the extant radio environment. Summarizing the results of the numerical experiments, the proposed model achieves the best forecasting performance.

\section{Conclusion}

This paper has introduced a blind auto regressive enhanced primary user emergence reasoning (AR-PUER) model for the cognitive radio. The method is based on linear prediction and case based reasoning. By using a combination of auto regressive prediction and primary user emergence reasoning, time similarity and time freeness are computed in our method. This is useful since it is desirable for a sensing model to operate without requiring the knowledge of the noise statistics. The time similarity is, thus, an indicator of the presence of the primary users in the signal that received by the secondary user.

The analysis results show that the performance of the proposed model is better than the conventional manners for the detection of primary users. Besides, the simulation outcomes demonstrate that the AR-PUER model is effective and generates the most accurate forecasting of primary users occurrence in several cases.

\section{Acknowledgement}

This work was supported in part by the National Natural Science Foundation of China under Grant No. 61471066 and 61402476, National High Technology Research and Development Program of China under Grant No. 2011AA01A204, and the Fundamental Research Funds for the Central Universities. 


\section{References}

[1] P. Kolodzy, Spectrum Policy Task Force, Federal Communications Commission Technology Report Docket 40.4, pp.147-158, November 2002. Article (CrossRef Link)

[2] A. Jajszczyk, "Cognitive Wireless Communication Networks (E. Hossian, and V. Bhargava)," IEEE Communications Magazine, vol. 30, no. 10, pp.18, November, 2008.

Article (CrossRef Link)

[3] Naeem M, Khwaja A S, Anpalagan A, “Green Cooperative Cognitive Radio: A Multiobjective Optimization Paradigm,” IEEE Systems Journal, vol. 10, no. 1, pp. 240-250, November, 2017. Article (CrossRef Link)

[4] Ren J, Zhang Y, Zhang N, "Dynamic Channel Access to Improve Energy Efficiency in Cognitive Radio Sensor Networks,” IEEE Transactions on Wireless Communications, vol. 15, no. 5, pp. 3143-3156, June, 2016. Article (CrossRef Link)

[5] Liu X, Jia M, Tan X, "Threshold optimization of cooperative spectrum sensing in cognitive radio networks,” Radio Science, vol. 48, no. 1, pp. 23-32, February, 2016. Article (CrossRef Link)

[6] Bkassiny M, Jayaweera S K, Li Y, "Blind Cyclostationary Feature Detection Based Spectrum Sensing for Autonomous Self-Learning Cognitive Radios,” in Proc. of IEEE International Conf. on Communications Communications, pp. 1507-1511, 2012. Article (CrossRef Link)

[7] Liao Y, Song L, Han Z, "Full duplex cognitive radio: A new design paradigm for enhancing spectrum usage,” IEEE Communications Magazine, vol. 53, no. 5, pp. 138-145, June, 2015. Article (CrossRef Link)

[8] Mesodiakaki A, Adelantado F, Alonso L, "Performance Analysis of a Cognitive Radio Contention-Aware Channel Selection Algorithm," IEEE Transactions on Vehicular Technology, vol. 64, no. 5, pp. 1958-1972, June, 2016. Article (CrossRef Link)

[9] Sun H, Nallanathan A, Cui S, "Cooperative Wideband Spectrum Sensing Over Fading Channels," IEEE Transactions on Vehicular Technology, vol. 65, no. 3, pp. 1382-1394, April, 2016. Article (CrossRef Link)

[10] Liang Zhou, “QoE-Driven Delay Announcement for Cloud Mobile Media,” IEEE Transactions on Circuits and Systems for Video Technology, vol. 27, no. 1, pp. 84-94, February, 2017. Article (CrossRef Link)

[11] Liang Zhou, "On Data-Driven Delay Estimation for Media Cloud," IEEE Transactions on Multimedia, vol. 18, no. 5, pp. 905-915, June, 2016. Article (CrossRef Link)

[12] A. Sonnenschien and P. M. Fishman, "Radiometric detection of spread-spectrum signals in noise of uncertain power,” IEEE Transactions on Aerospace and Electronic Systems, vol. 28, no. 3, pp. 654-660, April, 1992. Article (CrossRef Link)

[13] Juell $\mathrm{P}$ and Paulson P, "Using reinforcement learning for similarity assessment in case-based systems,” IEEE Intelligent systems, vol. 18, no. 4, pp. 60-67, May, 2003. Article (CrossRef Link)

[14] Jeng B C and Liang T P, "Fuzzy Indexing and Retrieval in Case-Based Systems,” Expert Systems with Applications, vol. 88, no. 1, pp. 135-142, February, 1995. Article (CrossRef Link)

[15] G. H. Golub and C. F. Van Loan, Matrix Computations, MD: Johns Hopkins University Press, Baltimore, 1983.

[16] F. F. Digham, M.-S. Alouini and M. K. Simon, “On the Energy Detection of Unknown Signals Over Fading Channels,” IEEE Transactions on Communications, vol. 55, no. 1, pp. 21-24, February, 2007. Article (CrossRef Link)

[17] Ying-Chang. Liang, Yonghong Zeng, Edward C.Y. Peh and Anh Tuan Hoang, "Sensing-Throughput tradeoff for cognitive radio networks," IEEE Transactions on Wireless Communications, vol. 7, no. 4, pp. 5330-5335, May, 2008. Article (CrossRef Link) 


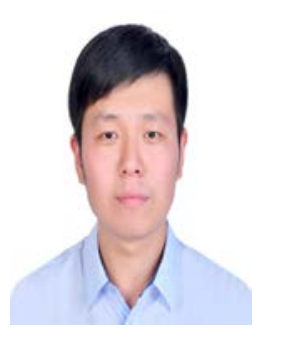

Wei Yang received the B.S. and M.S. degrees in 2011 and 2014, respectively, and he is currently working towards the Ph.D. degree in the field of Information and Communication Engineering at Beijing University of Posts and Telecommunications (BUPT). His research directions including wireless communication, information security and fusion.

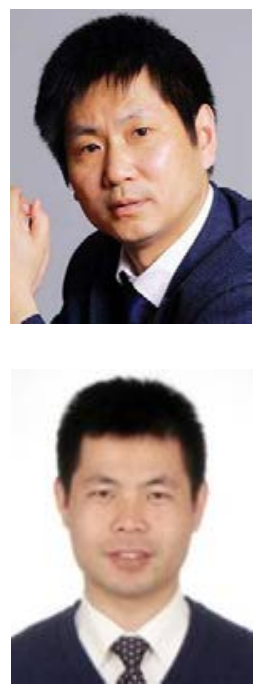

Xiaojun Jing received the bachelor's degree from the Beijing Normal University, and the M.S. and Ph.D. degrees from the National University of Defense Technology in 1995 and 1999, respectively. From 2000 to 2002, he was a Post-Doctoral Researcher with the Beijing University of Posts and Telecommunications (BUPT). He is a Full Professor with the School of Information and Communication Engineering, BUPT. His research directions including information security and fusion, wireless communication, and image processing.

Hai Huang received the Ph.D. degree from Beihang University, and he is a lecturer with the School of Information and Communication Engineering, Beijing University of Posts and Telecommunications (BUPT). His current research is related to intelligent information processing and virtual reality. 